Journal of Patient-Centered

\title{
In Adult Smokers Unwilling or Unable to Quit, Does Changing From Tobacco Cigarettes to Electronic Cigarettes Decrease the Incidence of Negative Health Effects Associated With Smoking Tobacco? A Clin-IQ
}

\author{
Jennifer Brown \\ Brandon Brown \\ Peter Schwiebert \\ Kalyanakrishnan Ramakrisnan \\ Laine H. McCarthy
}

Follow this and additional works at: https://aah.org/jpcrr

Part of the Medical Sciences Commons

\section{Recommended Citation}

Brown J, Brown B, Schwiebert P, Ramakrisnan K, McCarthy LH. In adult smokers unwilling or unable to quit, does changing from tobacco cigarettes to electronic cigarettes decrease the incidence of negative health effects associated with smoking tobacco? A Clin-IQ. J Patient Cent Res Rev. 2014;1:99-101. doi: 10.17294/2330-0698.1019

Published quarterly by Midwest-based health system Advocate Aurora Health and indexed in PubMed Central, the Journal of Patient-Centered Research and Reviews (JPCRR) is an open access, peer-reviewed medical journal focused on disseminating scholarly works devoted to improving patient-centered care practices, health outcomes, and the patient experience. 


\section{In Adult Smokers Unwilling or Unable to Quit, Does Changing From Tobacco Cigarettes to Electronic Cigarettes Decrease the Incidence of Negative Health Effects Associated With Smoking Tobacco? A Clin-IQ}

\section{Erratum}

This article mistakenly reports the delivery agent in electronic cigarettes as diethylene glycol instead of propylene glycol. Propylene glycol is a relatively benign substance commonly used in concert smoke machines. Diethylene glycol, a highly toxic substance, was reportedly found in only one e-cigarette cartridge studied in the literature, which may have been due to use of a nonpharmaceutical grade propylene glycol. JPCRR regrets this error. 


\title{
In Adult Smokers Unwilling or Unable to Quit, Does Changing From Tobacco Cigarettes to Electronic Cigarettes Decrease the Incidence of Negative Health Effects Associated With Smoking Tobacco? A Clin-IQ
}

\author{
Jennifer Brown, MD, Brandon Brown, MD, Peter Schwiebert, MD, Kalyanakrishnan Ramakrisnan, MD, \\ Laine H. McCarthy, MLIS \\ Family Medicine Residency Program, Department of Family \& Preventive Medicine, University of Oklahoma College of \\ Medicine, Oklahoma City, OK
}

\begin{abstract}
Data from a randomized controlled trial and systematic review support the claim that switching from tobacco cigarettes to electronic cigarettes (e-cigarettes) can reduce the short-term negative health effects of smoking. In adult smokers unwilling or unable to quit, exhaled carbon monoxide levels, total number of cigarettes smoked, and exposure to nitrosamine chemicals were reduced within a 12-month period. While the e-cigarette industry remains largely unregulated thus far, these studies provide encouraging hope in the uphill battle toward helping patients make informed and healthy choices. (J Patient-Centered Res Rev. 2014;1:99-101.)
\end{abstract}

\section{Keywords}

electronic cigarettes, e-cigarettes, tobacco, smoking, smoking cessation

\section{Search Terms}

adult, smoking, adverse effects of smoking, nicotine administration, nicotine dosage, smoking psychology, smoking cessation

\section{Clinical Question}

In adult smokers unwilling or unable to quit, does changing from tobacco cigarettes to electronic cigarettes decrease the negative health effects associated with smoking tobacco?

\section{Answer}

Probably, based on current evidence. More research is needed.

Date answer was determined: December 2013

Level of evidence for the answer: A

Correspondence: Laine H. McCarthy, MLIS, Clinical Associate Professor, Department of Family \& Preventive Medicine, University of Oklahoma College of Medicine, 900 NE 10th Street, Oklahoma City, OK, 73104, Phone: 405-271-8000, ext. 32206,

Email: laine-mccarthy@ouhsc.edu

\section{Inclusion Criteria}

Published systematic reviews, meta-analyses, randomized controlled trials and cohort studies comparing the shortterm and relatively long-term health effects of electronic cigarettes with tobacco cigarettes.

\section{Exclusion Criteria}

Pregnant women, children, and individuals with preexisting lung disease.

\section{Summary of the Issues}

Tobacco use is projected to kill 1 billion people in the 21 st century, making it the single greatest cause of preventable death globally. ${ }^{1}$ Tobacco use in any form has negative health consequences, the severity of which depends on the amount and duration of smoking as well as the type of nicotine delivery system employed. ${ }^{1}$ Tobacco cigarette smoke contains thousands of chemical byproducts (including 45 known or suspected carcinogens) that bind to DNA causing genetic mutations. Tobacco also contains nicotine, a highly addictive and psychoactive drug.

Electronic cigarettes (e-cigarettes) or electronic nicotine delivery systems, first introduced in China in 2006, have spread worldwide. ${ }^{2}$ Often made to resemble traditional cigarettes, e-cigarettes vaporize a solution containing nicotine and flavor in an agent such as diethylene glycol. The result is a relatively clean nicotine delivery system that should, in theory, have fewer adverse health effects when compared with traditional cigarettes. However, extensive data is lacking, and there remain significant concerns regarding the purity of the ingredients, device functionality, user modification and general lack of oversight regarding manufacturing and marketing. ${ }^{2}$

As popularity of e-cigarettes continues to increase, studies comparing the effects of e-cigarettes and traditional cigarettes on acute adverse health outcomes are emerging. ${ }^{3}$ This data is essential for recommending their use, particularly in smokers unwilling or unable to quit. The Jan. 8, 2014, tobacco-themed 
issue of The Journal of the American Medical Association focused on a number of different challenges that health care providers have encountered in the last 50 years regarding tobacco abuse and cessation attempts, and included a call for more research and regulation of e-cigarettes. ${ }^{4}$

\section{Summary of the Evidence}

Studies have shown that risks of these negative health effects are related to the length of time a person smokes and how much they smoke. ${ }^{1}$ As such, we chose to infer that reducing the extent and duration of tobacco cigarette smoking would, in turn, decrease these negative health effects. While more than 100 online and print publications were reviewed, only one randomized control trial was found that studied e-cigarette use in smokers unwilling or unable to quit. ${ }^{5}$ Various other publications that studied the safety of e-cigarettes referenced each other, and we chose to use information from two of the most comprehensive resources. ${ }^{3,5}$

Since long-term effects of e-cigarettes are unavailable, we chose to focus on measurable physiological and chemical parameters that have been scientifically linked to adverse health effects of smoking. Nitrosamine is a chemical compound found in latex, rubber, cosmetics and pesticides, as well as in tobacco smoke, snuff and snus, a moist powdery variation of snuff. ${ }^{3}$ More than $90 \%$ of nitrosamine derivatives are known to be highly carcinogenic and have been shown to cause a wide range of cancers. ${ }^{3}$ Carbon monoxide (CO), another byproduct of the combustion process, reduces the body's oxygen-carrying capacity and alveolar elasticity, promoting the development of emphysema and chronic obstructive pulmonary disease. ${ }^{3}$

Cahn et al., ${ }^{3}$ examining the evidence concerning the safety and efficacy of e-cigarettes, stated that only a minority $(5,300$ of the 10,000-100,000) of the chemicals in cigarette smoke have been detected, whereas all components in e-cigarette vapor have been identified using gas chromatography and mass spectrometry. Only two substances in e-cigarettes (nitrosamines and diethylene glycol) are believed to have negative health effects. The researchers noted that maximal levels of nitrosamine in e-cigarettes are comparable to those in a nicotine patch and are much lower than in tobacco cigarettes (Table 1). ${ }^{3}$ Diethylene glycol is a relatively benign substance commonly used in concert smoke machines, but it also has been associated with lethal toxicity at high levels. ${ }^{6}$

Caponnetto et al. ${ }^{5}$ designed a 12-month, prospective, randomized controlled trial to evaluate smoking reduction and/or abstinence among 300 smokers unwilling to quit using two different nicotine strength e-cigarettes: 2 groups
Table 1. Nitrosamine levels in various nicotine delivery systems ${ }^{3}$

\begin{tabular}{|l|l|}
\hline Nicotine delivery systems & $\begin{array}{l}\text { Nitrosamine } \\
\text { level }^{*}\end{array}$ \\
\hline Nicotine gum $(4 \mathrm{mg})$ & 2.00 \\
\hline Nicotine patch $(4 \mathrm{mg})$ & 8.00 \\
\hline Electronic cigarettes & 8.18 \\
\hline Snus (moist powdery smokeless tobacco) & $\sim 2,010$ \\
\hline Light cigarettes & $\sim 4,800$ \\
\hline Full-strength cigarettes & $\sim 6,200$ \\
\hline Dipping or smokeless tobacco (straight long cut) & $\sim 9,300$ \\
\hline
\end{tabular}

*Levels are reported as total nanograms in 1 gram of product.

$(\mathrm{n}=100$ each) received nicotine cartridges of different strengths; the third group $(\mathrm{n}=100)$ received non-nicotine cartridges for 12 weeks. Participants were not required to change their current smoking habits.

Over the 12-month period, nine follow-up visits were used to determine number of cigarettes smoked daily and exhaled $\mathrm{CO}$ levels. Both parameters decreased in the groups using e-cigarettes (Figure 1). A significant number of "reducers" (smokers who reduced daily cigarette use by more than $50 \%$ ) and "quitters" (those abstaining from smoking and had exhaled CO level $<7$ ppm) did not experience side effects commonly associated with quitting tobacco smoking. Rates of hunger $(6.5 \%)$, insomnia (4\%), irritability $(3.5 \%)$, anxiety $(3 \%)$ and depression $(2 \%)$ were lower than that expected with traditional tobacco smoking cessation methods. ${ }^{4}$ In addition, no weight gain was reported during this study.

Even though it is a relatively clean nicotine delivery system, e-cigarettes still contain nicotine. Nicotine is an addictive and psychoactive agent that causes physical dependence and tolerance while enhancing attention, concentration

Figure 1. Amount of exhaled carbon monoxide (in parts per million) and total cigarettes per day in electronic cigarette users that are unwilling to quit tobacco cigarette use. ${ }^{5}$

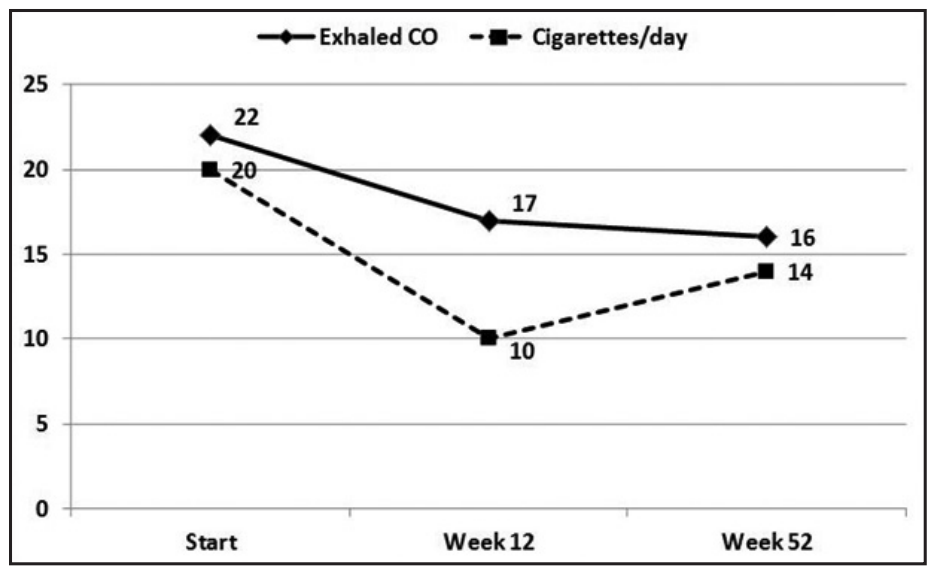


and mood. It is also a sympathomimetic drug that releases catecholamines, increasing heart rate and blood pressure, constricting blood vessels and reducing sensitivity to insulin. ${ }^{1}$ Nicotine thus has its own negative health effects, but long-term use of nicotine replacement products (gum, patch, etc.) have been shown to be generally well tolerated without evidence of serious adverse consequences. ${ }^{1}$

\section{Conclusion}

In tobacco smokers unwilling to quit, switching to e-cigarettes decreases exhaled $\mathrm{CO}$ level and number of tobacco cigarettes smoked. Furthermore, users of e-cigarettes are exposed to less carcinogenic nitrosamine than tobacco cigarette smokers. A reduction in exposure to $\mathrm{CO}$ and nitrosamines, as well as an overall reduction in smoking, helps reduce smoking-related negative health effects.

However, further studies are needed to evaluate the longand short-term effects of the diethylene glycol chemicals used as delivery agents in e-cigarettes. Improved product regulation and standardization of nicotine levels, safety, use and commercialization are required before e-cigarettes can be called a "safe" alternative to tobacco cigarette smoking. ${ }^{4}$ In the meantime, these early studies shed exciting new information on an addiction that has defied traditional treatment approaches.

\section{Acknowledgments}

Clin-IQ (Clinical Inquiry) is a required resident/faculty scholarly activity that takes questions from community practitioners and feeds the answers back out to improve patient care. L.H.M. received support from Oklahoma Shared Clinical \& Translational Resources, funded by grant number NIGMS U54GM104938, NIGMS/NIH.

\section{Conflicts of Interest}

None

\section{References}

1. Henningfield J, Hammond K; World Health Organization. Tobacco: deadly in any form or disguise. http://www.who.int/tobacco/ communications/events/wntd/2006/Report_v8_4May06.pdf. Accessed March 25, 2011.

2. O'Connor RJ. Non-cigarette tobacco products: what have we learnt and where are we headed? Tob Control. 2012;21:181-90.

3. Cahn Z, Siegel M. Electronic cigarettes as a harm reduction strategy for tobacco control: a step forward or a repeat of past mistakes? J Public Health Policy. 2011;32:16-31

4. Special theme issue of Journal of the American Medical Association: 50 Years of Tobacco Control. JAMA. 2014;311:145-212.

5. Caponnetto P, Campagna D, Cibella F, et al. EffiCiency and Safety of an eLectronic cigAreTte (ECLAT) as tobacco cigarettes substitute: a prospective 12-month randomized control design study. PLoS One. 2013;8:e66317.

6. Sosa NR, Rodriguez GM, Schier JG, Sejvar JJ. Clinical, Laboratory, Diagnostic, and Histopathologic Features of Diethylene Glycol Poisoning-Panama, 2006. Ann Emerg Med. 2014 Jan 15 [Epub ahead of print].

(C) 2014 Aurora Health Care, Inc. 